\title{
Evaluación de dos modelos de pisos trampa para el control del ácaro Varroa destructor Oud. sobre la abeja Apis mellifera L.
}

\author{
Evaluation of two models of floor traps for the control of the mite \\ Varroa destructor Oud. on bees Apis mellifera $L$. \\ Ximena Araneda Durán ${ }^{1}$, Alejandro Calzadilla Albornoz ${ }^{1}$
}

\begin{abstract}
RESUMEN
El ensayo se llevó a cabo a partir del 18 de agosto al 30 de diciembre de 2004 en el Apiario Experimental y en el Laboratorio de Sanidad de la Universidad Católica de Temuco ubicado en el Campus Norte Rudecindo Ortega 02950 en la ciudad de Temuco. El objetivo del estudio fue evaluar el uso de dos pisos trampa antivarroa (AV) como método de control físico para el ácaro Varroa destructor. Para ello se utilizaron 10 colonias de abejas Apis mellifera en colmenas Langstroth, con dos modelos de pisos trampa AV: cinco pisos trampa antivarroa modelo de tubos (AVT) (T1) y cinco pisos trampa antivarroa modelo de malla (AVM) (T2), los que fueron ubicados en la parte inferior de las colmenas. Las evaluaciones consistieron en la observación y recuento diario de ácaros Varroa caídos sobre las láminas del fondo de los pisos AV de ambos tratamientos, identificando vivos y muertos a fin de compararlos y determinar la eficacia de cada uno de los modelos de piso. Las eficacias determinadas fueron de 19,76\% y 15,87\% para los pisos trampa AV de malla y de tubo respectivamente. Ambos constituyen una alternativa de control físico para el ácaro varroa y pueden ser parte de una estrategia de control integrado del ácaro.
\end{abstract}

Palabras clave: Varroa destructor, pisos trampa antivarroa, Apis mellifera.

\begin{abstract}
The study was carried out between 18 August and 30 December 2004 in the Experimental Apiary and in the Health Laboratory of the Catholic University of Temuco, located in Campus Norte, Rudecindo Ortega 02950 in the city of Temuco. The aim of the study was to evaluate the use of anti-varroa (AV) floor traps as a method for the physical control of the mite Varroa destructor. The study was carried out on ten colonies of Apis mellifera bees in Langstroth hives, with two models of AV floor traps: five tube model anti-varroa floor traps $(A V T)(T 1)$ and five mesh model anti-varroa floor traps (AVM) (T2), which were placed in the bottoms of the hives. The evaluation consisted of the observation and daily count of varroa mites which fell onto the base sheets of the AV floor traps with each treatment, identifying live and dead mites in order to make a comparison and determine the effectiveness of each type of trap. The efficiencies determined for the mesh and tube AV traps were $19.76 \%$ and $15.87 \%$, respectively. Both types are options for the physical control of the varroa mite and may form part of an integrated mite control strategy.
\end{abstract}

Key words: Varroa destructor, anti-varroa floor traps, Apis mellifera.

\section{Introducción}

La varroasis causada por el ácaro Varroa destructor (Anderson, 2000; Anderson y Trueman, 2000) es una parasitosis que afecta a las abejas adultas y a sus crías causando serias pérdidas en la producción apícola del mundo (De la Sota y Bacci, 2004; CONASA, 2006). Para detectar la varroasis y la evaluación de su población en las colmenas es importante identificar el ácaro (Goodwin y Van Eaton, 2001), y así obtener una estimación precisa del tamaño de la población de varroas para la aplicación de prácticas de control integrado adecuadas, por lo que es necesario conocer con exactitud los métodos de muestreo utilizados (Branco et al., 2006). El control químico de Varroa, aunque pueda ofrecer una solución temporal a los apicultores, no constituye una solución a largo plazo (Vandame et al., 2002; Bogdanov, 2006). El uso intensivo de plaguicidas químicos puede generar resistencia, dejar residuos químicos, son costosos y podrían no ser accesibles para todos los apicultores.

Ningún método es totalmente efectivo para controlar la Varroa. Sin embargo, varias técnicas pueden ayudar a mantener la infestación de Varroa a un nivel manejable (Berkelaar et al., 2002).

1 Universidad Católica de Temuco, Campus Norte, Rudecindo Ortega 02950, Temuco, Casilla 15-D. E-mail: xaraneda@uct.cl

Fecha de Recepción: 31 Diciembre, 2008.

Fecha de Aceptación: 08 Marzo, 2011. 
Algunas opciones recomendadas por CONASA (2004) incluyen: el uso de cuadros o paneles trampa de zánganos y pisos trampa para varroas. Entre los pisos trampa se han propuesto varios diseños como método de control de Varroa (Berkelaar $e t$ al., 2002). Este control de la varroasis implica cambiar el piso de la colmena por una superficie con aberturas donde los ácaros caen y no pueden regresar a incorporarse a la colonia ni a las abejas en particular (Sandorf, 1998). Existen diferentes modelos de pisos trampa; uno de éstos consiste en un piso móvil de madera dotado de fondo con rejilla $(3 \mathrm{~mm})$ o malla de acero inoxidable, que permite la caída de los ácaros hasta unas bandejas donde son recogidos, impidiendo el acceso de las abejas (Shimanuki y Knox, 2000; De la Sota y Bacci, 2004; Brouard, 2005; Flores, 2007). Esta caída es probable que sea causada o favorecida por las abejas, las cuales son capaces de detectar, retirar y eliminar los ácaros ya sea sobre sí mismas (autogrooming) o sobre otras abejas (alogrooming) a través del comportamiento de acicalamiento (Flores et al., 1998; Moncada, 2004; Danka y Villa, 2005).

También existe el modelo de piso trampa de tubos donde las colmenas son equipadas con tubos de plástico que conforman el piso de la colmena. Este modelo está compuesto de 12 tubos que corren longitudinalmente con un espacio entre ellos para favorecer la caída del ácaro, pero a través de los cuales las abejas no pueden pasar (Le Pabic, 2002; Brouard, 2005; Jan-Baptiste, 2007). Este modelo fue inventado en 1993 por el apicultor Marcel Legris, y su objetivo fue reproducir las mismas condiciones observadas en muchas colonias silvestres de abejas en donde el ácaro no sobrevivía al caer en zonas no frecuentadas por las abejas (Le Pabic, 2002).

En este contexto, resulta importante evaluar la eficacia de dos modelos de piso trampa antivarroa (AV) en base a la reducción de las poblaciones de varroa que pueden ser alcanzadas con el uso de estos dispositivos.

\section{Materiales y Métodos}

El ensayo se llevó a cabo en el Apiario Experimental y en el Laboratorio de Sanidad de la Universidad Católica de Temuco ubicado en el Campus Norte Rudecindo Ortega 02950 en la ciudad de Temuco, a partir del 18 de agosto al 30 de diciembre de 2004.
Se utilizaron 10 colonias de abejas Apis mellifera en colmenas Langstroth. Se evaluó el uso de dos modelos de piso trama antivarroa (AV). Los tratamientos fueron $\mathrm{T} 1$ : cinco pisos trampa antivarroa de tubos (AVT) y T2: cinco pisos trampa antivarroa de malla (AVM).

Estos pisos AV fueron colocados en la parte inferior de las 10 colmenas, a las que previamente se les retiró el piso convencional. En ambos modelos de pisos se adaptó un fondo cerrado de madera, para localizar una lámina metálica impregnada con una película de vaselina líquida para fijar y contar diariamente la caída de los ácaros vivos y muertos.

Para el cálculo de la eficacia de ambos modelos de pisos, tubo y malla, se consideraron las varroas caídas durante el mes de octubre como el periodo para determinar y cuantificar el factor de corrección que equivale a aquellas varroas que caen de los pisos trampa pero que están muertas por alguna razón distinta al tratamiento y que por lo tanto deben ser restadas. Los datos de las varroas caídas en el mes de noviembre se consideraron como las equivalentes a los tratamientos y las varroas caídas en el mes de diciembre, como aquéllas producto del tratamiento químico (Bayvarol) que permite comparar el tratamiento contra la cantidad total de varroas presentes en las colonias.

La eficacia media del Bayvarol se define como 99,69\% según el fabricante (Bayer). De esta manera se obtiene en 30 días la caída total de los ácaros presentes en la colonia. El cálculo de la eficacia está dado por la fórmula adaptada de Flores et al. (1996).

A pasar de haber iniciado el ensayo en agosto solo se utilizaron los datos a partir de octubre, ya aquí se comenzó a registrar la presencia de varroas caídas vivas, las cuales podrían ser atribuidas a la presencia de los pisos trampa y por lo tanto evaluar su funcionamiento.

Se utilizó el programa estadístico SPSS. Versión 10.0 y la prueba de análisis estadística no paramétrica Kruskal-Wallis $(\mathrm{p} \leq 0,05)$.

\section{Resultados y Discusión}

La prueba de análisis estadística no paramétrica Kruskal-Wallis entre los tratamientos T1 y T2 entregó un $\alpha=0,478$ ( $p>0,05)$, donde no existieron diferencias estadísticamente significativas al comparar la caída promedio por día de varroas vivas entre ambos modelos de pisos trampa AV (Cuadro 1). 
Cuadro 1. Caída promedio por día de varroa para ambos tratamientos

\begin{tabular}{lccc}
\hline Modelo de piso trampa & Media & $\begin{array}{c}\text { Desviación } \\
\text { estándar }\end{array}$ & $\begin{array}{c}\text { Error } \\
\text { experimental }\end{array}$ \\
\hline Piso trampa AV tubo & 6,627 a & $\pm 4,2106$ & 0,7687 \\
Pisto trampa AV malla & 5,613 a & $\pm 2,8643$ & 0,5229 \\
\hline
\end{tabular}

Valores con letras iguales indican que no hay diferencias estadísticamente significativas según la prueba estadística no paramétrica de Kruskal Wallis 0,478 al 5\%.

Según Jan-Baptiste (2007) en evaluaciones realizadas en Francia, señala que las caídas más importantes de varroa ocurrieron el año 2007 sobre pisos trampa de tubo (AVT) con un 4,42\% de infestación, mientras que en los pisos trampa con malla la infestación aumentó a $6,13 \%$, por lo tanto el porcentaje de infestación en el piso AVT fue inferior al 5\% (Brouard, 2005).

En el presente estudio, la observación de la caída diaria de varroas permite tener una estimación de tamaño de la población del ácaro y por lo tanto inferir un nivel de infestación más confiable que el porcentaje de varroas presentes en abejas adultas, además permite determinar la fecha en que estos ácaros comienzan a caer vivos, lo que coincide con el mayor desarrollo y crecimiento de la colonia. En contraste, Eberhardt (2006), evaluando la aplicación de un producto de control de varroa no encontró correlación entre la caída natural y el nivel de infestación de la colmena para ninguno de los periodos evaluados (pre y postaplicación), señalando que la caída diaria de varroas no puede ser utilizada como un indicador del nivel de infestación de varroa en colmenas de la Región Metropolitana en Chile. Calderone (1999) indica que no existe una relación entre el porcentaje de infestación y la caída de ácaros, ya que colmenas con un mismo porcentaje de infestación pueden tener una distinta población de abejas, lo que varía el número de parásitos. Pese a esto Imdorf et al. (2003) señalan que la caída natural de varroa puede ser un buen indicador del tamaño de su población dentro de la colmena al relacionar la caída natural de varroa con la mortalidad de los ácaros posterior a un tratamiento con un varroacida.

Sin embargo, Martin (2006) señala que la caída natural del ácaro no asegura una disminución en la población de varroa, ya que puede generar una reinfestación al no existir una trampa en el piso de la colmena. No obstante, indica que la caída natural de varroa, representada por el testigo (uso de piso trampa modelo de malla), puede numérica y estadísticamente ser igual a la caída producida por efecto de otros tratamientos varroacidas, pero las consecuencias de su acción son diferentes, señalando que el método utilizado es adecuado para cuantificar el efecto de derribe de varroa pero no permite la determinación por sí solo del efecto acaricida del producto.

Además, se puede mencionar que el aumento en la caída de varroa viva y capturada en los pisos trampa AV, tanto de tubo como de malla durante el mes de noviembre del 2004, permite señalar que este mecanismo puede ejercer un control físico sobre el ácaro y su descendencia potencial, especialmente en el inicio de la primavera donde aumenta el desarrollo poblacional de las abejas y del ácaro varroa (Vandame, 2000). Con las temperaturas más altas se genera una mayor agitación dentro de las colmenas, un mayor roce entre las abejas, entre abejas y las pantallas de los pisos trampa AV, provocando el aumento en la caída de varroa viva capturada en ambos modelos pisos trampa AV. En este sentido, Melinor (2004) indica que los pisos de tubos promueven en teoría la mantención de las temperaturas internas óptimas de las colmenas y de acuerdo a lo señalado por Sammataro et al. (1999), generando una mayor agitación de las abejas con una mayor caída de las varroas, dicho comportamiento podría también ser válido para los pisos de malla, ya que la atmósfera interna de la colmena es controlada por el movimiento de las alas de las abejas, produciendo una circulación del aire, regulando así la temperatura y humedad (Pérez, 2007). Tal como lo mencionan Neira et al. (2004), quienes presentaron diferencias significativas en el desprendimiento de ácaros cuando las condiciones de temperatura, humedad y oscuridad se mantuvieron estables en el desarrollo de su ensayo.

De esta forma los pisos trampa antivarroa son considerados como una medida de control de la varroasis y se utilizan a menudo como parte de un manejo integrado de plagas, contribuyendo a disminuir las tasas de infestación en las colmenas al ejercer un control físico sobre el ácaro, proporcionando estimaciones precisas de las poblaciones de ácaros, reduciendo el número de ácaros, pero no proporcionan suficiente control sin la adición de otras medidas de control (Goodwin y Van Eaton, 2001). Tal como lo señalan Webster et al. (2000), y Chapleau (2003), quienes afirman que el uso de piso trampa AV o cualquier otro método que evite el regreso de los ácaros caídos a la colmena, parece también aumentar la eficacia 
de tratamientos varroacidas, ya que su uso puede retrasar el desarrollo de resistencia de $V$. destructor a los tratamientos químicos y permitir la sustitución de productos químicos acaricidas menos peligrosos que se utilizan actualmente, $y$ reducir naturalmente la varroa utilizando períodos prolongados de tiempo como indicadores del nivel de infestación de la colonia.

Al no existir diferencias estadísticamente significativas en relación a la caída de varroas en los modelos de piso trampa (AV), la decisión de compra o fabricación de uno u otro modelo debiera ser tomada en términos económicos, y según la realidad y las ventajas comparativas que posea cada empresa apícola. Los pisos trampa son de fácil uso, aplicables en cualquier época del año, y presentan los mejores resultados en la estación de otoño (Goodwin y Van Eaton, 2001).

Durante el periodo de evaluación se reportó que el mínimo de varroas caídas por día para ambos tratamientos fue de 1,6; mientras que el mayor número de ácaros caídos por día fue 18,2 y 12 varroas para los tratamientos AVT y AVM respectivamente.

Por su parte, Flores et al. (1998), en un trabajo realizado en España, reportaron que el número total de parásitos caídos durante doce controles en un conjunto de colonias ascendió a 786, de los cuales 375 (47,71\%) fueron varroas vivas, y los restantes $411(52,29 \%)$ estaban muertas. Mientras que Moncada (2004) en una evaluación realizada en el Sur de Chile encontró un total de 2.591 individuos caídos, de los cuales 8 $(0,31 \%)$ fueron varroas vivas, y los restantes 2.583 (99,69\%) estaban muertos; sin embargo, el conteo de varroas caídas no se hacía diariamente, por lo que esta estimación podría alterar la viabilidad de los ácaros al morir a causa de su caída sobre la vaselina líquida que los retenía.

\section{Eficacia del uso de piso trampa}

Posterior a la aplicación de Bayvarol ${ }^{\circledR}$ se procedió a contar la caída de varroas durante 30 días, para obtener el total de los ácaros por colmena. Según De Ruijter y Van den Eijnde (1991) el Bayvarol tendría una eficacia 99,69\%. Los resultados arrojaron un total de 5.431 varroas en el piso trampa AVT y 3.553 varroas para el piso trampa AVM, con lo cual se obtuvo una eficacia de un $15,87 \%$ y $19,76 \%$ para los pisos trampa AV de tubo y de malla respectivamente. Estos valores son semejantes a los señalados por Chapleau (2003), quien menciona que el uso de pisos trampa de mallas se basa en la observación de que el $20 \%$ de los ácaros causantes de la varroasis se caen de las abejas adultas a la parte inferior de la colonia y no pueden volver a la abeja, debido a la estructura de la malla, produciéndose la muerte del ácaro.

En el presente estudio, ambos porcentajes de eficacia $(15,87 \%$ y $19,76 \%)$ son importantes considerando que provienen de un método físico, que puede ser sostenido en el tiempo, no deja residuos y no causa resistencia, a diferencia de las experiencias con los tratamientos químicos, como lo indican Webster et al. (2000), quienes señalan que la resistencia de este ácaro al acaricida es quizás el problema más serio que enfrentan los apicultores en Europa y América del Norte. Asimismo, el uso intensivo de plaguicidas químicos puede generar resistencia cruzada en los ácaros, tal es el caso de los piretroides sintéticos fluvalinato y flumetrina (Goodwin y Van Eaton, 2001; Wang et al., 2002). Además, otro de los problemas con los productos sintéticos es que en su mayoría presentan consecuencias adversas de contaminación de miel, cera y polen (Calderone y Spivak, 1995).

Además, ambos porcentajes de eficacia reportados en este estudio son menores que los resultados obtenidos en pisos trampa AVM por Clark (1999), quien señala un 25\% a 60\%; Webster et al. (2000), $39 \%$ al 50\%; Chapleau (2003), 37\%; con la excepción de Pettis y Shimanuki (1999), quienes mencionan un $14 \%$ y $28 \%$ para los meses de junio y julio.

\section{Conclusiones}

El piso de trampa AVM presenta una eficacia aproximada al 20\% con relación al 16\% de eficacia del piso trampa AVT, no existiendo diferencias estadísticamente significativas en relación a la caída de varroas en ambos modelos. Luego la decisión de compra o fabricación de uno u otro modelo debiera ser tomada en términos económicos, y según la realidad y las ventajas comparativas que posea cada empresa apícola

Los pisos trampa AV tanto de tubo como de malla constituyen una alternativa de control físico para el ácaro varroa siendo permanente en el tiempo y de fácil uso, evitan la resistencia de los ácaros y pueden formar parte de estrategias de manejos integrados para el control de varroa. 


\section{Literatura Citada}

Anderson, D.L.

2000 Variation in the parasitic bee mite Varroa jacobsoni Oud. Apidologie, 31:281-292.

Anderson, D. and Trueman, J.

2000 Varroa jacobsoni (Acari: Varroidae) is more than one species. Experimental and Applied Acarology 24(3):165-189.

Berkelaar, D.; Davis, K.; Cox, D.

2002 Control de garrapatas en abejas melíferas. ECHO Notas de Desarrollo EDN. $N^{\circ} 73$ p. 1-5.

Bogdanov, S.

2006 Contaminants of bee products. Apidologie 37:1-18.

Branco, M.; Kidd, N.; Pickard, R.

2006 A comparative evaluation of sampling methods for Varroa destructor (Acari: Varroidae) population estimation. Apidologie 37:452-461.

Brouard, I.

2005 Trial report on the Happykeeper bottom board. Exploitation du Lycée agricole de Toulouse-Auzeville. E.P.L.E.A. Toulouse-Auzeville. 25 p.

Calderone, $\mathrm{N}$.

1999 Evaluation of formic acid and thymol based bled of natural products for the fall control of Varroa jacobsoni (Acari: Varroidae) in colonies of Apis mellifera (Hymenoptera: Apidae). Journal of Economic Entomology (USA) 92(2):253-260.

Calderone, N. and Spivak, M.

1995 Plant extracts for control of the parasitic mite Varroa jacobsoni (Acari: Varroidae) in colonies of the western honey bee (Hymenoptera: Apidae). Journal of Economic Entomology (USA) 88(5):1211-1215.

Chapleau, J.P.

2003 Experimentation of an Anti-Varroa screened bottom board in the context of developing an integrated pest management strategy for Varroa infested honeybees in the Province of Quebec. Ministère de l'Agriculture, des Pêcheries et de l'Alimentaiton du Québec, Canada. 19 p.

Clark, K.

1999 Evaluation of mesh Bottom Boards for the Management of Varroa Mites. [en línea]: Canadian Honey Council. Documento electrónico obtenido de internet. [fecha de consulta: 20 de junio 2004]. Disponible en: http://www. honeycouncil.ca/ users/folder.asp@ FolderID=4924.htm.

CONASA

2004 Recomendaciones para el Control de Varroa. Revista Actualidad Apícola (Uruguay) $\mathrm{N}^{\circ}$ 84, pp. 62-63.

CONASA

2006 Recomendaciones para el control de varroasis. Programa de Control de Enfermedades de las Abejas. SENASA. Argentina. $8 \mathrm{p}$.

Danka, R. and Villa, J.

$2005 \mathrm{An}$ association in honey bees between autogrooming and the presence of migrating tracheal mites. Apidologie 36:331-333.

De la Sota, M. y Bacci, M.

2004 Enfermedades de las abejas. Manual de procedimientos. Trámites en apicultura. Servicio Nacional de Sanidad y Calidad Agroalimentaria (SENASA). Dirección Nacional de Sanidad Animal. Buenos Aires. 55 p.
De Ruijter, A.; Van Den Eijnde, J.

1991 Ensayo de campo para determinar la eficacia de las tiras ("strips") de Bayvarol contra los ácaros varroa en colonias de abejas, así como la acción del preparado sobre el desarrollo de la colonia en los meses siguientes a la aplicación. Not. Med.- Vet. 61:30-35.

Eberhardt, C.

2006 Caída natural de Varroa destructor Anderson \& Trueman (Mesostigmata: Varroidae), como indicador del nivel de infestación y efecto del tratamiento otoñal con ácido fórmico sobre la colmena en la Región Metropolitana, Chile. Tesis (Ingeniero Agrónomo). Valdivia, Región de Los Ríos, Chile, Universidad Austral de Chile, Facultad de Ciencias Agrarias, Escuela de Agronomía. 68 p.

Flores, J.

2007 Selección de abejas tolerantes a Varroa. IX Jornada Malagueña de Apicultura. Antequera, 10 de febrero de 2007. Asociación de Apicultores Guadalhorce de Málaga. 7 p.

Flores, J.M.; Ruiz, J.A.; Ruz, J.M.; Puerta, F.; Campano, F.

1996 Avances en el estudio de los tratamientos naturales contra Varroa jacobsoni. II Congreso de la Sociedad Española de Agricultura Ecológica. Agricultura Ecológica y Desarrollo Rural. Pamplona-Iruña, septiembre de 1996. pp. 511-517.

Flores, J.M.; Ruiz, J.A.; Ruz, J.M.; Puerta, F.; Campano, F.; Padilla, F.; Bustos, M.

1998 El grooming en Apis mellifera Ibérica frente a Varroa jacobsoni Oud. Archivos de Zootecnia 47(178-179):213-218.

Goodwin, M. and Van Eaton, C.

2001 Control of varroa. A guide for New Zealand Beekeepers. New Zealand Ministry of Agriculture and Forestry (MAF). Wellington, New Zealand. $120 \mathrm{p}$.

Imdorf, A.; Charriere, J.; Kilchenmann, V.; Bogdanov, S.; Fluri, P.

2003 Alternative strategy in central Europe for the control of Varroa destructor in honey bee colonies. Apiacta (Rumania) 38:258-285.

Jan-Baptiste, M.

2007 Essais sur le plateau de fond de ruche a tubes "Happykeeper". Centre de Formation Professionnelle et de Promotion Agricoles. Les formations aux métiers du vivant. EPLEFPA de Vesoul. Le Grand Montmarin. Franche Comité Consell regional. $11 \mathrm{p}$.

Le Pabic, J.P.

2002 Présentation du plateau à tubes Happykeeper. [en línea]. HAPPYKEEPER. Documento electrónico obtenido de internet. [fecha de consulta: 10 de octubre 200]. Disponible en http://www.beekeeping.com/happykeeper/ index_fr.htm\#article

Martin, F.

2006 Evaluación de la aplicación estival de Apilife Var ® en el control de Varroa destructor Anderson y Trueman, ectoparásito de Apis mellifera L. Tesis (Ingeniero Agrónomo). Valdivia, Región de Los Ríos, Chile. Universidad Austral de Chile, Facultad de Ciencias Agrarias, Escuela de Agronomía. 109 p.

Melinor.

2004 Entre abejas, piso inteligente Melinor. Revista del Pequeño y Mediano Apicultor 1(1):10-11.

Moncada, M.S.

2004 Evaluación del comportamiento de acicalamiento (grooming) de abejas Apis mellifera L. con relación al ácaro 
Varroa jacobsoni Oud. En la comuna de Padres Las Casas, IX Región. Tesis (Ingeniero Agrónomo). Temuco, Región de La Araucanía, Chile, Universidad Católica de Temuco, Facultad de Ciencias Agropecuarias y Forestales, Escuela de Agronomía. 73 p.

Neira, M.; Heinsohn, P.; Carrillo, R.; Báez, A.; Fuentealba, J. 2004 Efecto de aceites esenciales de lavanda y laurel sobre el ácaro Varroa destructor Anderson \& Trueman (Acari:Varroidae). Agricultura técnica 64 (3): 238-244.

Pérez, $\mathrm{H}$.

2007 Evaluación del efecto del ácido fórmico sobre Varroa destructor Anderson \& Trueman (Mesostigmata: Varroidae), aplicado en otoño, sobre colonias de Apis mellifera L. (Hym: Apidae) en Valdivia. Tesis (Ingeniero Agrónomo). Valdivia, Región de Los Ríos, Chile, Universidad Austral de Chile, Facultad de Ciencias Agrarias, Escuela de Agronomía. 74 p.

Pettis, J.S. and Shimanuki, H.

1999 A hive modification to reduce varroa populations. American Bee Journal 139 (6): 471-473.

Sammataro, D.; Finley, J.; Camazine, S.

1999 Shipping conditions of honey bee queens. American Bee Journal 139 (9): 713-716.

Sandorf, M.T.

1998 The "sticky board": a new apicultural tool. APIS 16(5): 3.

Shimanuki, H. and Knox, D.

2000 Diagnosis of honey bee diseases. United States Department of Agriculture (USDA), Agricultural Research Service, Agriculture Handbook No 690. Beltsville, USA. 57 p.
Vandame, R.

2000 Control alternativo de varroa en apicultura. [en línea]: El colegio de la Frontera Sur. Proyecto "abejas de Chiapas". Edición 2.2. Documento electrónico obtenido de internet. [Fecha de consulta: 27 septiembre 2004]. Disponible en: https://ssl.domicile.fr/apiservices.com/articulos/control_varroa/curso2.htm

Vandame, R.; Colin, M.; Otero, G.

2002 Abejas europeas y africanizadas en México: la tolerancia a Varroa jacobsoni. [en línea] Primera parte: Biología de Varroa. México. Documento electrónico obtenido de internet. [fecha de consulta: 10 de octubre 2008]. Disponible en: http:// www.culturaapicola.com.ar/apuntes/sanidad/233_abejas $\% 20$ tolerancia1.pdf

Wang, R.; Liu, Z.; Dong, K.; Elzen, P.; Pettis, J.; Huang, Z. 2002 Association of novel mutations in a sodium channel gene with fluvalinate resistance in the mite, Varroa destructor. Journal of Apicultural Research (Inglaterra) 40(1-2): 17-25. Webster, T.C.; Thacker, E.M.; Vorisek, F.E.

2000 Live Varroa jacobsoni (Mesostigmata: Varroidae) fallen from honey bee (Hymenoptera: Apidae) colonies. Entomological Society of American 93 (6): 1596-1601. 